\title{
PREVALENCE OF NON-ALCOHOLIC FATTY LIVER DISEASE AND ITS CORRELATION WITH CORONARY RISK FACTORS IN PATIENTS WITH TYPE 2 DIABETES MELLITUS
}

Sheela Krishna Murthy ${ }^{1}$, G. N. Nagesh ${ }^{2}$

\section{HOW TO CITE THIS ARTICLE:}

Sheela Krishna Murthy, G. N. Nagesh. "Prevalence of Non-Alcoholic Fatty Liver Disease and its Correlation with Coronary Risk Factors in Patients with type 2 Diabetes Mellitus". Journal of Evolution of Medical and Dental Sciences 2015; Vol. 4, Issue 47, June 11; Page: 8101-8107, D0I:10.14260/jemds/2015/1174

ABSTRACT: Nonalcoholic fatty liver disease (NAFLD) is one of the most common causes of chronic liver disease. It encompasses a spectrum of conditions associated with lipid deposition in hepatocytes. It ranges from steatosis (Simple fatty liver), to nonalcoholic steatohepatitis (NASH-fatty changes with inflammation and hepatocellular injury or fibrosis), to advanced fibrosis and cirrhosis. Studies suggest that although simple fatty liver is a benign condition, NASH can progress to fibrosis and lead to end-stage liver disease. The disease is mostly silent and is often discovered through incidentally elevated liver enzyme levels. It is strongly associated with obesity and insulin resistance and is currently considered by many as the hepatic component of the metabolic syndrome. NASH cirrhosis is now one of the leading indications for liver transplantation in the United States. Accurate epidemiologic data in India is not available because of a lack of population-based studies and reliable non-invasive screening tools. There is disagreement about the methods used to diagnose NASH, and there is no clear consensus on the clinical implications of histologic changes. The prevalence of NAFLD is affected by many factors, including genetics and environment and is therefore difficult to define. In general, the risk of liver disease increases with the patient's body.

KEYWORDS: NAFLD, Insulin resistance, Type 2 diabetes.

INTRODUCTION: Non-alcoholic fatty liver disease (NAFLD) is a common condition characterised by excess of fat in liver which ranges from simple steatosis to steatohepatitis, cirrhosis and hepatocellular carcinoma (HCC) in the absence of excessive alcohol intake.[1] Metabolic syndrome and conditions associated with it like diabetes, obesity and dyslipidemia are predisposing factors of NAFLD. ${ }^{[2]}$ NAFLD is becoming a major public health problem due to rising incidence of obesity and type II diabetes.[3] The overall prevalence of NAFLD is 15 to $40 \%$ in western countries while $9-40 \%$ in Asian countries.[1] The data from Asian countries derived from various published series where there was no uniformity in definition of NAFLD and population studied. NAFLD is a common cause of chronic liver disease and liver transplantation in western countries. ${ }^{[4]}$ Increasing incidence of NAFLD has been well documented from Asian countries like Japan and China.[5] Diabetes mellitus (DM), obesity, hyperinsulinemia are predisposing factors NAFLD. There is increase in incidence of DM, obesity and insulin resistance in India in last two decades. ${ }^{[6,7]}$ Hence it is logical to expect increase in incidence of NAFLD in India. There is limited data on the prevalence of NAFLD from India.[8,9] Therefore this study was planned to estimate the prevalence of NAFLD in general population.

The pathogenetic processes of nonalcoholic fatty liver disease (NAFLD) and its progression are multifactorial and are influenced by both environmental and genetic factors.[9,10]

Although the mechanism is complex and incompletely understood, a 2-hit hypothesis has been proposed[11] in which the first hit involves an imbalance of fatty acid metabolism that leads to 


\section{ORIGINAL ARTICLE}

hepatic triglyceride accumulation (Steatosis). The second hit may be oxidative or metabolic stress and dysregulated cytokine production resulting from efforts to compensate for altered lipid homeostasis, leading to subsequent inflammation and fibrosis. Data show that hepatic mitochondrial dysfunction is crucial to the pathogenesis of NAFLD.[12]

Nonalcoholic fatty liver disease (NAFLD) is one of the most common causes of chronic liver disease. It encompasses a spectrum of conditions associated with lipid deposition in hepatocytes. It ranges from steatosis (Simple fatty liver), to nonalcoholic steatohepatitis (NASH-fatty changes with inflammation and hepatocellular injury or fibrosis), to advanced fibrosis and cirrhosis. Studies suggest that although simple fatty liver is a benign condition, NASH can progress to fibrosis and lead to end-stage liver disease. The disease is mostly silent and is often discovered through incidentally elevated liver enzyme levels.[13] It is strongly associated with obesity and insulin resistance and is currently considered by many as the hepatic component of the metabolic syndrome. NASH cirrhosis is now one of the leading indications for liver transplantation in the United States. Accurate epidemiologic data in India is not available because of a lack of population-based studies and reliable noninvasive screening tools. There is disagreement about the methods used to diagnose NASH, and there is no clear consensus on the clinical implications of histologic change.[14] The prevalence of NAFLD is affected by many factors, including genetics and environment and is therefore difficult to define. In general, the risk of liver disease increases with the patient's body mass.

The metabolic syndrome is not an absolute risk indicator, because it does not contain many of the factors that determine absolute risk, for example, age, sex, cigarette smoking, and low-density lipoprotein cholesterol levels.[15] Nonetheless, patients with the metabolic syndrome are at twice the risk of developing CVD over the next 5 to 10 years as individuals without the syndrome. The risk over a lifetime undoubtedly is even higher. Furthermore, the metabolic syndrome confers a 5 -fold increase in risk for type 2 diabetes mellitus. The most widely recognized of the metabolic risk factors are atherogenic dyslipidemia, elevated blood pressure, and elevated plasma glucose. In addition, persons with these characteristics commonly manifest a prothrombotic state and a proinflammatory state.

A number of studies suggest that postprandial or post-load glucose and postprandial triglyceride concentrations are more strongly associated with some of the CVD risk factors and mortality than the respective fasting values. ${ }^{[16-18]}$ They debate the causal role of postprandial glucose in relation to CVD,[19] versus simply reflecting the underlying metabolic abnormalities in for example lipid metabolism. It has also been suggested that the relation between hyperglycemia and CVD is mediated by oxidative stress.[20]

OBJECTIVES: To estimate the prevalence of non-alcoholic fatty liver disease (NAFLD) by ultrasonography, and to correlate NAFLD with coronary artery disease (CAD) and coronary risk factors in a group of Indian type 2 diabetics.

METHODOLOGY: Consecutive patients of type 2 diabetes were recruited. History and physical examination were recorded.

Laboratory investigations included fasting blood glucose, liver function tests, lipid profile and glycated haemoglobin. NAFLD was diagnosed on the basis of ultrasound assessment of the liver.

RESULTS: The study group of Type 2 diabetic patients $(n=109)$ was divided into a NAFLD group $(n=64)$ and a non-NAFLD group $(n=45)$. The prevalence of NAFLD was 65\%. 
CAD was more prevalent in the NAFLD subgroup (15\%) compared to the non-NAFLD subgroup (13\%). The NAFLD subgroup had higher prevalence of hypertension, smoking, obesity (Measured by BMI), central obesity (Measured by waist circumference and waist hip ratio), higher HbA1c, higher triglyceride levels and lower HDL levels.

Using the Mann-Whitney test, it was found that BMI $(\mathrm{p}=0.022)$ correlated statistically to NAFLD, metabolic syndrome and CAD.

DISCUSSION: A total of 109 patients (64 men and 45 women) with type 2 diabetes were included. The prevalence of NAFLD was $65 \%$, with men having a higher prevalence $(56.3 \%)$ as compared to women (43.7\%). Fatty liver showed a bimodal peak with a male predominance.

More than half of the study population was obese and dyslipidemic, as almost $53.5 \%$ and $57.9 \%$ had $\mathrm{BMI}>25 \mathrm{~kg} / \mathrm{m} 2$ and serum triglycerides $>150 \mathrm{mg} / \mathrm{dl}$, respectively. The prevalence of obesity (BMI>25kg/m2) in patients with NAFLD was $53.5 \%$, as compared to $46.5 \%$ in non-NAFLD patients. CAD was more prevalent in the NAFLD subgroup (15\%) as compared to the non-NAFLD subgroup (13\%).

On analyzing the risk factors for CAD, the NAFLD subgroup had a higher prevalence of hypertension, smoking, obesity (Measured by BMI), central obesity (Measured by waist circumference and WHR), higher HbA1c and triglyceride levels and lower HDL level.

Using a cut off level of $\mathrm{HbA1c}>7 \%$ as a measure for poor control, $89 \%$ in the non-NAFLD subgroup and $85 \%$ in the NAFLD group had poor glycaemic control (Odds ratio=0.87). Metabolic syndrome, as defined by IDF (2005) criteria, was present in $65 \%$ of the study group.

The NCEP, ATP III definition of the metabolic syndrome is based on simple clinical and biochemical parameters, while other available definitions of the metabolic syndrome include measures which are expensive and difficult to measure in developing countries.

There is increasing belief that NCEP, ATP III definition of the metabolic syndrome is not optimal for the identification of risks for T2DM or CHD, and does not identify the metabolic syndrome correctly in South Asians. Most important limitation is that the internationally accepted cut-off points of waist circumference (men $>102 \mathrm{~cm}$, and women, $>88 \mathrm{~cm}$ ) for diagnosis of abdominal obesity are not applicable for South Asians. This is supported by our recent data that show that waist circumference levels of $>90 \mathrm{~cm}$ and $>80 \mathrm{~cm}$ for men and women, respectively, were associated with high odds ratios for the presence of cardiovascular risk factor(s).[21-22]

Recently, International Diabetes Federation (IDF) recommended a new definition of the metabolic syndrome. This definition included three major modifications as compared to NCEP, ATP III definition. [23]

Prevalence of the metabolic syndrome was significantly higher in the NAFLD subgroup, as compared to those who did not have NAFLD (61.9\% vs. 13.2\%). Mean values of liver enzymes (AST and ALT) were higher in the NAFLD subgroup. Patients with higher degree of liver steatosis disease had poorer glycaemic control and greater derangements in lipid profile. However, the metabolic syndrome was equally present in both subgroups. Liver enzymes were shigher in higher degrees of NAFLD ( $p=0.156)$. In the study done by A.K Agarwal et al, it was found that hypertension $(p=0.013)$, LDL cholesterol $(p=0.049)$, microalbuminuria $(p=0.034)$ and NAFLD $(p=0.016)$ were independent predictors of CAD.

A number of studies have found a positive relationship between hyperinsulinaemia, abnormal glucose tolerance, and NAFLD.[24] Mishra et al found the prevalence of metabolic syndrome and 


\section{ORIGINAL ARTICLE}

NAFLD to be $24 \%$ and $14.8 \%$, respectively, in non-alcoholic North Indian men. In a study by Mohan et al the prevalence of NAFLD (54.5\%) was significantly higher in patients with diabetes compared to those with pre-diabetes (IGT or IFG) (33\%), isolated IGT (32.4\%), isolated IFG (27.3\%) and normal glucose tolerance (NGT) (22.5\%). Also in this study, it was found the prevalence of most cardiometabolic risk factors was significantly higher in NAFLD patients. Gupta et al found that mild, moderate, and severe NAFLD was present in $65.5 \%, 12.5 \%$, and $9.35 \%$ of otherwise asymptomatic type 2 diabetics, respectively. Prashanth et al found a high prevalence of NAFLD and NASH in type 2 diabetics which increased with multiple components of the metabolic syndrome. Banerjee et al observed that, on histology, only fatty change was present in $43 \%$, NASH in $40 \%$ and more advanced disease in $23 \% .^{[25-26]}$

In our study, the prevalence of NAFLD, as detected by ultrasound, was $65 \%$ which is comparable with the prevalence found in other studies (Gupta et al, Prashanth et al, Banerjee et al). As seen in other studies, mean total cholesterol and LDL levels did not correlate with NAFLD; however there was a significant correlation with high triglyceride and low HDL levels. Kessler et al showed that the prevalence of NAFLD, as diagnosed by ultrasound, was significantly higher in patients with acute myocardial infarction compared with that found in the general population; moreover, NAFLD was associated with greater severity of coronary artery disease independent of age, sex and body mass index.[27-28]

A limitation of our study is that the diagnosis of NAFLD was based on ultrasonography and was not confirmed by liver biopsy. Ultrasonography is by far the commonest method of diagnosing NAFLD in clinical practice and has very good sensitivity and specificity. The sensitivity and specificity of ultrasound for detecting hepatic steatosis varies from 60 to $94 \%$ and 88 to $95 \%$, respectively.

\section{Studies suggest that liver biopsy is seldom necessary to diagnose NAFLD:}

- Central obesity has been made a mandatory variable,

- The cut-offs of waist circumference have been lowered (male, $94 \mathrm{~cm}$; female, $80 \mathrm{~cm}$ ), and for south Asians: (Male, $90 \mathrm{~cm}$; female, $80 \mathrm{~cm}$ ),

- Cut-off level for fasting plasma glucose has been lowered to $100 \mathrm{mg} / \mathrm{dl}$.

According to a recent study on south Indians, the prevalence of the metabolic syndrome (\%) was estimated to be 23.2, 18.3 and 25.8 according to the WHO, ATPIII and IDF definitions respectively.

Prevalence of the metabolic syndrome was significantly higher in the NAFLD subgroup, as compared to those who did not have NAFLD (61.9\% vs. 13.2\%). Mean values of liver enzymes (AST and ALT) were higher in the NAFLD subgroup. Patients with higher degree of liver steatosis disease had poorer glycaemic control and greater derangements in lipid profile. However, the metabolic syndrome was equally present in both subgroups. Liver enzymes were shigher in higher degrees of NAFLD ( $p=0.156)$. In the study done by A.K Agarwal et al, it was found that hypertension $(p=0.013)$, LDL cholesterol $(p=0.049)$, microalbuminuria $(p=0.034)$ and NAFLD $(p=0.016)$ were independent predictors of CAD.

Is NAFLD a new risk factor for cardiovascular disease? A significant number of studies have related, in several forms, the NAFLD with cardiovascular disease. Nevertheless, limitations mainly related to design, sample and method of diagnosing, makes it hard to have a definitive conclusion. Prognostic studies showed significantly increased mortality in patients with NAFLD compared to the 
general population. In these studies, cardiovascular disease was the leading or at least the second leading cause of death, featuring an increased risk of death from cardiovascular disease in patients with NAFLD. However, most of these patients had overweight or obesity, impaired fasting glucose or impaired glucose tolerance or diabetes, hypertension and metabolic syndrome.[29-35]

\section{REFERENCES:}

1. Farrell GC, Larter CZ. Nonalcoholic Fatty Liver Disease: from Steatosis to cirrhosis. Hepatology 2006; 43: S00-S112.

2. McCullough AJ. Thiazolidinediones for nonalcoholic steatohepatitis-promising but not ready for prime time. N Engl J Med 2006; 355 (22): 2361-3.

3. Yoon KH, Lee JH, Kim JW, Cho JH, Ko SH, Zimmet P, San H Y. Epidemic obesity and type 2 diabetes in Asia. Lancet 2006; 368: 1681-1688.

4. Clark JM. Epidemiology of Nonalcoholic Fatty Liver Disease in Adults. J Clin Gastroenterol 2006; 40 (Suppl 1): S5-S10.

5. Amarapurkar DN, Hashimoto E, Lesmana LA, Sollano JD, Chen P, Goh KL, for the Asia-Pacific Working Party on NAFLD. How Common is NAFLD in the Asia-Pacific Region, and are There Local Differences? J Gastroenterol Hepatol 2007; 22: 788-93.

6. Mohan V, Deepa R. Adipocytokines and The expanding «Asian Indian Phenotype». J Assoc Physic Ind 2006; 54: 685-6.

7. Misra A, Vikram NK. Insulin resistance syndrome (metabolic syndrome) and Asian Indian. Current Science 2002; 83: 1483-96.

8. Singh SP, Nayak S, Swain M, et al. Prevalence of nonalcoholic fatty liver disease in coastal eastern India; a preliminary ultrasonographic survey. Trop Gastroenterol 2004; 25: 76-9.

9. Duseja A, Chawla Y. Nonalcoholic fatty liver disease in India How much? How Soon? Trop Gastroenterol 2005; 2.

10. Ludwig J, Viggiano TR, McGill DB, Oh BJ. Nonalcoholic steatohepatitis: Mayo Clinic experiences with a hitherto unnamed disease. Mayo Clin Proc. Jul 2010; 55 (7): 434-8.

11. Chitturi S, Farrell GC, Hashimoto E, Saibara T, Lau GK, Sollano JD. Non-alcoholic fatty liver disease in the Asia-Pacific region: definitions and overview of proposed guidelines. J Gastroenterol Hepatol. Jun 2007; 22 (6): 778-87.

12. Browning JD, Szczepaniak LS, Dobbins R, et al. Prevalence of hepatic steatosis in an urban population in the United States: impact of ethnicity. Hepatology. Dec 2004; 40 (6): 1387-95.

13. Weston SR, Leyden W, Murphy R, Bass NM, Bell BP, Manos MM, et al. Racial and ethnic distribution of nonalcoholic fatty liver in persons with newly diagnosed chronic liver disease. Hepatology. Feb 2005; 41 (2): 372-9.

14. Clark JM. The epidemiology of nonalcoholic fatty liver disease in adults. J Clin Gastroenterol. Mar 2006; 40 Suppl 1: S5-10.

15. Younossi ZM. Review article: current management of non-alcoholic fatty liver disease and nonalcoholic steatohepatitis. Aliment Pharmacol Ther. Jul 2008; 28 (1): 2-12.

16. Delgado JS. Evolving trends in nonalcoholic fatty liver disease. Eur J Intern Med. Mar 2008; 19 (2): 75-82.

17. Patton HM, Sirlin C, Behling C, Middleton M, Schwimmer JB, Lavine JE. Pediatric nonalcoholic fatty liver disease: a critical appraisal of current data and implications for future research. J Pediatr Gastroenterol Nutr. Oct 2006; 43 (4): 413-27. 


\section{ORIGINAL ARTICLE}

18. Kim $\mathrm{CH}$, Younossi ZM. Nonalcoholic fatty liver disease: a manifestation of the metabolic syndrome. Cleve Clin J Med. Oct 2008; 75 (10): 721-8.

19. Rector RS, Thyfault JP, Wei Y, Ibdah JA. Non-alcoholic fatty liver disease and the metabolic syndrome: an update. World J Gastroenterol. Jan 14 2008; 14 (2): 185-92.

20. Jou J, Choi SS, Diehl AM. Mechanisms of disease progression in nonalcoholic fatty liver disease. Semin Liver Dis. Nov 2008; 28 (4): 370-9.

21. Wei Y, Rector RS, Thyfault JP, Ibdah JA. Nonalcoholic fatty liver disease and mitochondrial dysfunction. World J Gastroenterol. Jan 14 2008; 14 (2): 193-9.

22. Palasciano G, Moschetta A, Palmieri VO, et al. Non-alcoholic fatty liver disease in the metabolic syndrome. Curr Pharm Des. 2007; 13 (21): 2193-8.

23. Adams LA, Angulo P, Lindor KD. Nonalcoholic fatty liver disease. CMAJ. Mar 29 2005; 172 (7): 899-905.

24. Erickson SK. Nonalcoholic fatty liver disease. J Lipid Res. Apr 2009; 50 Suppl: S412-6.

25. Jiang J, Torok N. Nonalcoholic steatohepatitis and the metabolic syndrome. Metab Syndr Relat Disord. Mar 2008; 6 (1): 1-7.

26. Lindor KD, on behalf of the UDCA/NASH Study Group. Ursodeoxycholic acid for treatment of nonalcoholic steatohepatitis: results of a randomized, placebo-controlled trial [abstract]. Gastroenterology. 2003; 124 (4): A708-A709.

27. Oh MK, Winn J, Poordad F. Review article: diagnosis and treatment of non-alcoholic fatty liver disease. Aliment Pharmacol Ther. Sep 1 2008; 28 (5): 503-22.

28. Schwimmer JB, Behling C, Newbury R, et al. Histopathology of pediatric nonalcoholic fatty liver disease. Hepatology. Sep 2005; 42 (3): 641-9.

29. Yeh MM, Brunt EM. Pathology of nonalcoholic fatty liver disease. Am J Clin Pathol. Nov 2007; 128 (5): 837-47.

30. Kleiner DE, Brunt EM, Van Natta M, et al. Design and validation of a histological scoring system for nonalcoholic fatty liver disease. Hepatology. Jun 2005; 41 (6): 1313-21.

31. Matteoni CA, Younossi ZM, Gramlich T, Boparai N, Liu YC, McCullough AJ. Nonalcoholic fatty liver disease: a spectrum of clinical and pathological severity. Gastroenterology. Jun 1999; 116 (6): 1413-9.

32. Brunt EM, Kleiner DE, Wilson LA, et al. Portal chronic inflammation in nonalcoholic fatty liver disease (NAFLD): a histologic marker of advanced NAFLD-Clinicopathologic correlations from the nonalcoholic steatohepatitis clinical research network. Hepatology. Mar 2009; 49 (3): 80920.

33. Lackner C, Gogg-Kamerer M, Zatloukal K, Stumptner C, Brunt EM, Denk H. Ballooned hepatocytes in steatohepatitis: the value of keratin immunohistochemistry for diagnosis. J Hepatol. May 2008; 48 (5): 821-8.

34. Angulo P. Long-term mortality in nonalcoholic fatty liver disease: is liver histology of any prognostic significance? Hepatology. Feb 2010; 51 (2): 373-5.

35. Day CP. Natural history of NAFLD: remarkably benign in the absence of cirrhosis. Gastroenterology. Jul 2005; 129 (1): 375-8. 


\section{ORIGINAL ARTICLE}

\section{AUTHORS:}

1. Sheela Krishna Murthy

2. G. N. Nagesh

\section{PARTICULARS OF CONTRIBUTORS:}

1. Assistant Professor, Department of Internal Medicine, BGS Institute of Medical Science, Bangalore.

2. Professor \& Unit Chief, Department of Internal Medicine, KIMS, Bangalore.

\section{FINANCIAL OR OTHER}

COMPETING INTERESTS: None

NAME ADDRESS EMAIL ID OF THE

CORRESPONDING AUTHOR:

Dr. Sheela Krishna Murthy, BGS GIMS Hospital,

Uttarahalli, Bangalore.

E-mail: sheelakm@gmail.com

Date of Submission: 24/02/2015. Date of Peer Review: 21/04/2015. Date of Acceptance: 04/06/2015. Date of Publishing: 09/06/2015. 